\title{
Caráter estigmatizante da tuberculose, natureza biológica e impacto social da doença
}

\author{
Stigmatizing Character Of Tuberculosis, Its Biological Nature And Social Impact \\ ÉRIKA FELIX da Silva SANTOS. \\ Aluna Graduanda do curso de Odontologia da Universidade \\ Estadual da Paraíba. Campina Grande/PB, Brasil. \\ Anna Kássia Tavares Alves Chaves Santiago. \\ Aluna Graduanda do curso de Odontologia da Universidade \\ Estadual da Paraíba. Campina Grande/PB, Brasil. \\ Douglas Pereira de Sousa. \\ Aluno Graduando do curso de Odontologia da Universidade \\ Estadual da Paraíba. Campina Grande/PB, Brasil. \\ Bruno Alisson Freire Pedrosa. \\ Graduando do curso de Odontologia da Universidade \\ Estadual da Paraíba. Campina Grande/ PB, Brasil.
}

Verônica Felix da Silva Santos. Graduanda do curso de Enfermagem da Faculdade Maurício de Nassau. Campina Grande/ PB, Brasil.

Maria Helena Chaves de Vasconcelos Catão. Professora Doutora do Departamento de Odontologia, do curso de Odontologia da Universidade Estadual da Paraíba, Campina Grande/PB, Brasil.

\begin{abstract}
RESUMO
Introdução:ATuberculose, causada pela espécie Mycobacterium tuberculosis, é uma doença infectocontagiosa que causa reações inflamatórias intensas nos tecidos acometidos. Está presente comumente entre populações pobres, porém apresenta distribuição mundial, sendo considerada, desde 1993, pela Organização Mundial da Saúde (OMS), a primeira doença prioritária de saúde pública mundial. A farmacoterapia complexa utilizada no tratamento é um grande empecilho para controle da doença, que é transmitida por meio de gotículas de saliva contendo o agente infectante. Objetivo: $O$ presente estudo tem por objetivo geral refletir a respeito dos mecanismos que possibilitaram à tuberculose interferir na saúde do ser humano após tanto tempo desde sua descoberta. Material e métodos: Para isto, foi realizada uma revisão bibliográfica de cunho qualitativo, desenvolvida com base em publicações científicas disponíveis nos bancos de dados Scielo e Pubmed, assim como em livros de microbiologia e patologia, manuais e relatórios do Ministério da Saúde e da OMS. Conclusão: O histórico da doença tem mostrado melhoras em relação a outros anos, porém, para seu controle, é necessário optar por um modelo de saúde humanizado, que articule a participação social e o envolvimento intrínseco dos profissionais de saúde e poderes públicos mundiais.
\end{abstract}

Palavras-chave: TUberCulose, SAÚde pública, Mycobacterium tUBerCulosis.

\begin{abstract}
Introduction: Tuberculosis, caused by Mycobacterium tuberculosis, is an infectious disease that causes severe inflammatory reactions in the affected tissues. It is commonly present in poor populations, however, it has a worldwide distribution and is considered by the WHO (World Health Organization), since 1993, the first priority disease in global public health. The complex pharmacotherapy used in the treatment is a major obstacle to control the disease that is transmitted through droplets of saliva containing the infectious agent,
\end{abstract}

FOL • Faculdade de Odontologia de Lins/Unimep • 24(1) 41-50 • jan.-jun. 2014

ISSN Impresso: 0104-7582 • ISSN Eletrônico: 2238-1236

DOI: http://dx.doi.org/10.15600/2238-1236/fol.v24n1p41-50 
making thousands of victims every year. Objective: This study aims at reflecting on the general mechanisms that allowed tuberculosis to interfere in the human health so much time since its discovery. Material and methods: To this end, a literature review of qualitative nature was developed based on scientific publications available in Scielo and Pubmed databases, as well as books of microbiology and pathology, manuals and reports from the Ministry of Health and WHO. Conclusion: The disease's profile has greatly improved over the years, however, its control demands that a humanized model of health is chosen, encompassing social participation and the intrinsic involvement of health professionals and world public powers.

Keywords: Tuberculosis, Public health, Mycobacterium tuberculosis.

\section{INTRODUÇÃO}

A tuberculose é uma doença infectocontagiosa causada por micobactérias que promovem reações inflamatórias intensas nos tecidos à sua volta. $O$ órgão mais afetado é o pulmão, em virtude da afinidade do microorganismo com o oxigênio. ${ }^{1}$ Sua distribuição está diretamente relacionada às características socioeconômicas apresentadas pelas comunidades sociais às condições precárias de vida e ao descuido com a saúde. ${ }^{2}$

A tuberculose apresenta importante repercussão na cavidade oral, o que dificulta a diagnose pelos cirurgiões-dentistas. As lesões orais da tuberculose são incomuns; não está claro se elas evoluem da disseminação hematogênica ou por exposição à secreção catarral contaminada. $\mathrm{O}$ manejo terapêutico dessas lesões é feita por meio da terapia utilizada contra a tuberculose. ${ }^{3}$

É uma enfermidade de notificação compulsória e está inserida entre as doenças ocupacionais às quais os profissionais da saúde estão expostos. Nesse sentido, como os cirurgiões-dentistas são profissionais que lidam diariamente com secreções infectantes, a utilização dos equipamentos de proteção individual é fundamental em sua prevenção

Apesar de existirem grupos de risco aos quais são preconizadas medidas de prevenção, todas as pessoas são susceptíveis a desenvolver a infecção pelo bacilo. Por isso, é de suma importância que a população e os profissionais da saúde tenham conhecimento para planejamento, execução e análise de ações que visem ao controle da tuberculose no mundo.
Diante do exposto, o presente estudo tem por objetivo refletir a respeito dos mecanismos que possibilitam a permanência da tuberculose entre as doenças de interesse público, descrevendo enfoques históricos, epidemiológicos e aspectos relevantes no controle da infecção, bem como a participação das políticas de saúde pública na busca pela qualidade no tratamento e controle da doença.

\section{Metodologia}

A presente pesquisa foi realizada por meio de uma revisão bibliográfica de cunho qualitativo desenvolvida com base em dados presentes em publicações já elaboradas utilizando os seguintes termos: tuberculose, Mycobacterium tuberculosis; lesões orais da tuberculose; tuberculose como doença ocupacional; tuberculose e HIV; estratégia, saúde da família e tuberculose; tratamento da tuberculose; histórico da tuberculose.

A pesquisa de dados foi realizada, inicialmente, a partir da delimitação do tema para o estudo. Os materiais utilizados foram artigos científicos disponibilizados em revistas eletrônicas, no banco de dados Scielo e Pubmed, livros de microbiologia e patologia oral e maxilofacial, manuais e relatórios disponíveis no site do Ministério da Saúde e da Organização Mundial da Saúde (OMS).

Posteriormente, foi realizada uma análise concisa para identificar os dados que iriam compor o presente artigo, em busca da elaboração de um texto que conseguisse reunir as informações mais significativas a respeito da tuberculose, da situação atual desta doença 
no mundo e perspectivas futuras com relação ao controle e tratamento desta infecção.

\section{REVISÃo DE LITERATURA}

Tuberculose: a infecção depois de séculos

A tuberculose é causada por micobactérias pertencentes ao gênero Mycobacterium da espécie Mycobacterium tuberculosis. Ela foi descrita por Robert Koch em 1882, porém "o fato de ter sido detectada em múmias egípcias comprova que ela já comprometia o homem há 3.400 anos antes de Cristo". ${ }^{4}$

No decorrer do século XIX até metade do século $X X$, a tuberculose era considerada uma doença romântica, pois era comum entre artistas e intelectuais, muitos dos quais apresentavam estilo de vida boêmio, cujas obras relatavam aspectos negativos da infecção e o que sentiam perante a forma como era tratada na época. 5,6

A transmissão da tuberculose é feita de pessoa para pessoa por meio de gotículas de saliva contendo o agente infectante, ao respirar, falar, tossir. "O tuberculoso portador de doença cavitária em seu pulmão é a principal fonte de infecção e o responsável pela disseminação da doença em sua comunidade. Isso faz dele o principal objeto de atenção das ações de controle da tuberculose."4

Os sintomas mais frequentes da tuberculose pulmonar são: tosse que persiste por mais de duas semanas, que pode estar acompanhada de catarro e sangue; febre; sudorese; cansaço; dor no peito; falta de apetite; emagrecimento. ${ }^{4,7}$ Dependendo de fatores do hospedeiro, a tuberculose pode disseminar-se pelo organismo afetando outros órgãos nos quais os sintomas são mais específicos. ${ }^{7}$

A tuberculose pleural é a forma extrapulmonar mais comum; ela apresenta sintomas como dor torácica unilateral e falta de ar e há possibilidade de ocorrer derrame pleural. A tuberculose ganglionar tem como quadro clínico o aumento dos linfonodos na região do pescoço que tendem a agrupar-se e formar fístulas. A tuberculose óssea costuma envolver a coluna vertebral, causando destruição dos tecidos em volta e provocando dores que variam de leves a intensas. ${ }^{4}$

A tuberculose cerebral é a forma mais grave, muito comum em pessoas com sistema imune deprimido, porém representa uma pequena parcela dos casos de tuberculose em virtude da vacinação BCG. Esta forma de tuberculose provoca cefaleia, alteração do comportamento e nível de consciência, e pode evoluir como uma meningite tuberculosa ou com a formação de tuberculomas cerebrais. ${ }^{4,7}$

As manifestações bucais apresentamse como úlceras crônicas persistentes; menos frequentemente, podem surgir áreas leucoplásicas, sendo a linha média do dorso da língua, o palato e lábios os sítios mais prevalentes. Pode ocorrer o envolvimento dos nódulos linfáticos cervicais e da estrutura óssea da mandíbula e maxila, causando osteomielite tuberculosa, relatadas como áreas radiolúcidas mal definidas. ${ }^{3} \mathrm{O}$ envolvimento do côndilo mandibular é ainda mais raro, e a manifestação mais comum é um inchaço doloroso localizado na mandíbula. ${ }^{8}$

A importância do diagnóstico precoce em lesões orais pode ser um desafio para o cirurgião-dentista, especialmente porque essas lesões podem se comportar clinicamente como sinais de outras doenças mais comuns. ${ }^{9}$ O diagnóstico padrão para a tuberculose é a baciloscopia, desenvolvida há mais de cem anos, e cultura com identificação da espécie. A baciloscopia consiste na observação de bactérias em amostras de expectoração examinadas sob um microscópio; o problema deste exame é a demora do resultado. ${ }^{10}$

Outro exame que auxilia no diagnóstico da tuberculose é o teste de reatividade da pele, chamado de PPD (derivado de proteína purificado), ou exame de tuberculina. 
Este exame baseia-se na reação de hipersensibilidade. Se o paciente já foi exposto à bactéria, seu organismo possui anticorpos que atacarão as proteínas inoculadas na pele, resultando em reação inflamatória intensa no local da inoculação. O PPD só se torna positivo entre duas e dez semanas após o contágio., ${ }^{4,7}$

A prevenção da tuberculose inclui evitar aglomerações, locais fechados e mal ventilados e a imunização das crianças com a vacina BCG, que tem sido utilizada desde 1920 e faz parte do calendário nacional de vacinação. Porém, apesar de esta vacina prevenir contra os casos graves da doença, ela não evita a tuberculose por completo e não é indicada para ser administrada em crianças imunossuprimidas por serem feitas com bactérias vivas.

No Brasil, o tratamento é oferecido pelo Sistema Único de Saúde (SUS) e tem duração média de seis meses; inclui três medicações eficazes: rifampicina, isoniazida e pirazinamida. "No atual cenário da luta contra a tuberculose, um dos aspectos mais desafiadores é o abandono do tratamento, pois repercute no aumento dos índices de mortalidade, incidência e multidrogarresistência." 11

\section{DistRIBUIÇÃo DA TUBERCULOSE NO MUNDO: DADOS EPIDEMIOLÓGICOS}

A tuberculose foi a primeira doença a ser declarada questão prioritária de saúde pública mundial pela OMS, em 1993, porém continua sendo um problema global e considerada a segunda causa de morte por doenças infecciosas em todo mundo, depois do HIV.

Em 2011, foram quase 9 milhões de novos casos e 1,4 milhão de mortes por tuberculose, sendo 430 mil casos de morte associados à comorbidade com o HIV. Geograficamente, em 2009, o peso da tuberculose era maior na Ásia e África, dados que corroboram informações atuais da Organização Mundial da Saúde, ${ }^{11}$ que colocam a Índia e China correspondendo a quase $40 \%$ dos casos mundiais de tuberculose, sendo que $80 \%$ dos casos de tuberculose entre portadores do vírus HIV residem na África.

Desde 2003, essa doença é considerada prioridade pelo Governo Federal do Brasil, que ocupa a $13^{a}$ posição entre os 22 países que concentram $80 \%$ dos casos de tuberculose no mundo. ${ }^{5}$ A taxa de incidência, em 2001, foi de 42,8 casos para grupos de 100 mil habitantes, e caiu para 36 no ano de 2011. A região Sudeste concentra o maior número de casos de tuberculose, porém a região Norte apresentou maiores taxas de incidência em todos os anos analisados.

Em 2011, os estados do Amazonas $(62,6)$ e Rio de Janeiro $(57,6)$ apresentaram as maiores taxas de incidência do Brasil para cada grupo de 100 mil habitantes. Aproximadamente 66\% dos casos são de pessoas do sexo masculino, frequentemente afetando homens na faixa etária entre 45 e 54 anos de idade. A região Sul $(18,6)$ possui o maior percentual de casos de tuberculose em portadores do vírus HIV. Os estados de Santa Catarina $(21,1)$ e Rio Grande do Sul $(20,6)$ apresentaram as maiores de taxas percentuais de coinfecção com o vírus HIV. ${ }^{2}$

\section{HISTÓRICO}

O termo "tuberculose" foi criado em 1839 por Schoenlein, que aproveitou a palavra "tubérculo", nome dado por Sylvios Deleboe em 1680 ao nódulo lesional provocado pela infecção. As pessoas que apresentaram tuberculose até esta data eram chamadas de "tísicas". ${ }^{6}$

$\mathrm{Na}$ fase medieval da história havia dezenas de tuberculosos com formas graves disseminadas pelo corpo. Entre $O$ final do século XVIII e início do século XIX, a Revolução Industrial contribuiu bastante para agravar o quadro de disseminação da tuberculose em virtude das condições das cidades populosas, onde as pessoas viviam e trabalhavam em condições subumanas, morando em lugares pequenos e populosos. Nesta época, a tuberculose foi denominada 
a "grande praga branca", porque geralmente acometia populações caucasianas e também para diferenciá-la da peste bubônica. ${ }^{12}$

Nas conquistas territoriais, o homem civilizado levou o bacilo da tuberculose aos nativos, que, sem defesas imunitárias, tiveram grandes contingentes dizimados. ${ }^{6}$ Acredita-se que a tuberculose foi trazida ao Brasil pelos portugueses e missionários jesuítas a partir da colonização do País. ${ }^{13}$

Antes da quimioterapia medicamentosa e da descoberta do agente etiológico, as melhorias na habitação, saneamento básico e nutrição na Europa têm sido associadas à redução da tuberculose. ${ }^{14,15} \mathrm{~A}$ introdução de substâncias medicamentosas no controle da doença modificou consideravelmente sua história natural, possibilitando a diminuição da letalidade desta infecção. A quimioterapia veio romper a correlação estabelecida entre óbitos e número de casos presentes em dada comunidade. ${ }^{16}$

A descoberta dos fármacos utilizados no combate à tuberculose pode ser associada à penicilina, em 1928, que apesar de não ser tão eficaz no tratamento, ofereceu subsídios para estudos subsequentes. Após a descoberta da penicilina, Selmam Waksman descobriu, em 1944, a estreptomicina, que foi o primeiro antibiótico capaz de combater a tuberculose. Novos medicamentos foram utilizados com sucesso, destacando-se a isoniazida, em 1952; a rifampicina, em 1965; o estambutol, empregado em 1968, e a pirazinamida, usada em $1970 .{ }^{5}$

Apesar de a tuberculose ser uma doença antiga, existem atualmente poucos e antigos medicamentos utilizados em seu tratamento, apresentando diversos problemas, como efeitos indesejados, tratamento prolongado e surgimento de micro-organismos resistentes, sendo um importante problema de saúde pública mundial. No entanto, por ser uma doença considerada negligenciada, comum principalmente em países subdesenvolvidos e em desenvolvimento, a tuberculose tem recebido pouca atenção por parte das multinacionais e centro de pesquisas na busca e produção de novos medicamentos. ${ }^{5}$

A explicação para a falta de desenvolvimento de novos medicamentos é a carência de incentivos financeiros relacionados à pesquisa do tratamento de doenças que afetam principalmente a população pobre. ${ }^{15}$

\section{Mycobacterium tuberculosis: características gerais, patogenia e imunologia \\ Ogênero Mycobacterium consiste em bacilos} aeróbios imóveis que não formam esporos e medem de 0,2 a 0,6 $\mu \mathrm{m}$ de largura por 1 a $10 \mu \mathrm{m}$ de comprimento. As micobactérias apresentam parede celular complexa e são caracterizadas como micro-organismos fastidiosos. Seu crescimento é lento, requerendo um período de oito semanas para que sejam detectadas em cultura de laboratório. ${ }^{17}$

M. tuberculosis é aeróbio obrigatório, o que explica sua preferência por tecidos com grande concentração de oxigênio. No corpo, esse micro-organismo localiza-se, principalmente, no interior de células retículo-endoteliais, como os macrófagos, onde sobrevivem e multiplicamse no interior do vacúolo celular sintetizando uma proteína denominada "proteína repetitiva exportada", cuja ação impede a fusão do fagossomo com o lisossomo, permitindo que o micro-organismo escape das enzimas degradativas e sobreviva. ${ }^{18}$

Embora a fagocitose seja iniciada pelos macrófagos, outras células de defesa circulantes são atraídas para o local da infecção por meio de restos celulares, antígenos e substâncias quimiotáticas do hospedeiro que geram reações inflamatórias. As lesões mais comuns são a exsudativa e a granulomatosa. ${ }^{17,18}$

A reação inflamatória pode resultar em necrose caseosa, uma forma distinta da necrose de coagulação, frequentemente encontrada em focos de tuberculose. O termo 
"caseosa" deriva da aparência macroscópica de queijo branco da área de necrose. ${ }^{19}$

A replicação intracelular das micobactérias estimula células $T$ auxiliares (CD4+) e células $T$ citotóxicas. A resposta imune também leva à ativação de anticorpos, porém estes não são muito efetivos porque as micobactérias são protegidas no interior dos macrófagos. ${ }^{17}$

Em resposta à infecção por $M$. tuberculosis, os macrófagos liberam interleucinas e fator de necrose tumoral. Essas substâncias aumentam a reação inflamatória, recrutando outras células de defesa, como células exterminadoras naturais (NK). As células $\mathrm{T}$ auxiliadoras secretam interferon-g, levando ao aumento da fusão fagossomo-lisossomo e liberação de óxido nítrico e intermediário reativo. ${ }^{1,7}$

Macrófagos alveolares, células epitelioides e células gigantes de Langhans formam, com os bacilos intracelulares, uma massa central com tecido necrosado que é circundada por uma densa barreira de células T CD4, T CD8, NK e macrófagos. Esta estrutura recebe o nome de granuloma. ${ }^{17}$

Quando a resposta imune do paciente é ativada, a replicação do bacilo cessa, na maioria dos casos, entre três e seis semanas após a exposição ao micro-organismo. Cerca de $5 \%$ dos casos expostos a $M$. tuberculosis progridem para a doença; outros $5 \%$ a $10 \%$ apresentam a doença em algum momento da vida. ${ }^{17}$ A reativação da infecção pode ocorrer em resposta a perturbações no sistema imune, como a infecção pelo HIV, presença de diabetes mellitus, uso de corticosteroides, envelhecimento e abuso de drogas. ${ }^{20}$

\section{Condições agRaVAntes dA tUberculose: MULTIRRESISTÊNCIA E HIVI AIDS}

O termo tuberculose multirresistente (TB-MDR) foi cunhado nos Estados Unidos em 1980, quando surgiram formas clínicas resistentes à rifampicina e à isoniazida. A infecção é considerada multirresistente quando seu agente etiológico, a bactéria $M$. tuberculosis, apresenta resistência in vitro à rifampicina e à isoniazida e a mais um terceiro medicamento que compõe a farmacoterapia padronizada. ${ }^{10,21}$

A tuberculose multirresistente é atribuída a acumulações de mutações independentes nos genes do cromossomo da micobactéria. A causa do surgimento da multirresistência está relacionada a fenômenos iatrogênicos decorrentes do uso inadequado ou incorreto de antimicrobianos ou o uso de formulações de medicamentos ineficientes. ${ }^{10,21}$

Otratamento da tuberculose multirresistente é complicado, pois o paciente não responde aos medicamentos mais usados e poderosos contra a doença, sendo necessária a utilização de drogas mais caras e mais tóxicas ao organismo. $^{10} \mathrm{O}$ tratamento torna-se difícil na associação com o HIV, estando associado com alta taxa de mortalidade. ${ }^{22}$

A apresentação clínica da tuberculose em indivíduos infectados pelo HIV é influenciada pelo grau de imunossupressão. A tuberculose extrapulmonar é mais comum em pacientes com Aids, e a forma pulmonar é mais comum em portadores do vírus HIV. ${ }^{23}$

$\mathrm{O}$ vírus HIV compromete o sistema de defesa do corpo, favorecendo o desenvolvimento de doenças oportunistas. No caso da tuberculose, esta condição facilita a disseminação hematogênica do bacilo. Porém a tuberculose também influi no curso da infecção pelo HIV por meio da ativação de células de defesa infectadas pelo vírus, levando a um desenvolvimento precoce da Aids. ${ }^{4}$

Pacientes que possuem coinfecção tuberculose/HIV têm maior probabilidade de apresentar um desfecho desfavorável ao tratamento da tuberculose, que representa a primeira causa de morte em pacientes com Aids no Brasil. Por isso, o Ministério da Saúde recomenda que seja realizado o teste anti-HIV em todos os pacientes com tuberculose..$^{2,16}$ 


\section{Caráter estigmatizante da tuberculose:} IMPACTO SOCIAL E PSICOLÓGICO

A tuberculose provoca, entre outros problemas sociais, mudanças negativas, como o afastamento e isolamento na vida pessoal do doente. Enfrentar um diagnóstico positivo envolve sentimentos de medo, de vergonha e significa, para muitos, estar diante de manifestações de preconceitos, levando o paciente a esconder sua condição por receio da rejeição familiar e de amigos. ${ }^{24}$

Em geral, o diagnóstico positivo é um acontecimento estressante que ameaça a realidade da vida do paciente e promove seu isolamento do mundo e profunda alteração da autoestima. ${ }^{19}$ Essas mudanças internas que os pacientes enfrentam refletem-se em seu desempenho profissional. A ideia de ser demitido ou conviver com a imagem deteriorada pelos colegas reforça o sentimento de medo e de recusa ao exame diagnóstico, o que acaba por tornar-se um obstáculo ao tratamento. ${ }^{24}$

$\mathrm{O}$ afastamento de familiares e amigos contribui para o surgimento de pânico em relação à doença, como a separação dos utensílios, e apresenta-se como sentido ambivalente, pois, ao mesmo tempo em que essa atitude indica cuidado com o outro, também pode promover o preconceito. ${ }^{24}$

Sengupta et al., ${ }^{25} \mathrm{em}$ estudo realizado no sul da Tailândia, identificou termos que sugerem o estigma da doença, sendo os contaminados chamados de tra-bahb e tee-tra, que significam, respectivamente, "marcado com o pecado" e "carimbado", além das expressões "sujos" e "separados", podendo indicar preconceito religioso.

\section{Programas e estratégias para CONTROLE DA TUBERCULOSE}

Desde 1993, quando a tuberculose foi declarada estado de urgência, a OMS uniu forças com instituições de alto nível científico e poder econômico para criar o programa Stop TB. ${ }^{13}$
O programa Stop TB está entrelaçado com outras estratégias que têm como meta reduzir, em 2015, a prevalência e a mortalidade por tuberculose em 50\% em relação a 1990 e, em 2050 , eliminar esta doença como problema de saúde pública. Para isto, tem como objetivo, dentre outros, alcançar o acesso universal aos cuidados de alta qualidade para todos com tuberculose e, sobretudo, proteger a população vulnerável. ${ }^{10}$

Em 1998, dada a permanência do problema da tuberculose, foi lançado o Plano Nacional de Controle da Tuberculose. Este plano introduziu como novidades: a extensão da cobertura, o tratamento diretamente observado (Directly Observed Treatment Short-Course - Dots) e nova forma de repasse financeiro para os municípios. ${ }^{26,27}$

A estratégia Dots tem sido considerada exitosa no que se refere a mudanças significativas dos índices de cura da tuberculose, pois tem contribuído para a redução do abandono e da mortalidade. ${ }^{26,28}$

A Dots é constituída por cinco pilares: detecção de casos por baciloscopia; tratamento de curta duração observável e monitorado em sua evolução; fornecimento regular de medicamentos; sistema de registro e informação que assegure a avaliação do tratamento e monitoramento dos resultados e; compromisso político, colocando o controle da tuberculose como prioridade entre as políticas públicas de saúde. ${ }^{29,22}$

\section{Discussão}

A tuberculose está inserida entre as doenças de interesse para os cirurgiões-dentistas por ser uma doença infectocontagiosa sistêmica com repercussão na cavidade oral. Segundo Neville et al., ${ }^{3}$ a descoberta da tuberculose pulmonar ativa como resultado da investigação de lesões orais ocorre, mas não é comum. Já Wang et al. ${ }^{9}$ dizem que a tuberculose na região de cabeça e pescoço pode não ser tão rara como se pensa, mostrando a importância do 
diagnóstico diferencial de tuberculose para lesões ulcerativas lentas que não respondem ao tratamento convencional, pois tais lesões oriundas da tuberculose podem sugerir neoplasias malignas e acarretar uma série de expectativas negativas para profissional e paciente. Por isso, a biópsia e a investigação sistêmica apresentam papel importante no diagnóstico destas lesões.

O perfil de evolução da tuberculose tem melhorado em relação a outros anos, porém a doença permanece fazendo vítimas, e sua distribuição pelo mundo mantém-se quase inalterada no decorrer dos anos. Hargreaves et al. ${ }^{28}$ reforçam que o progresso no controle da tuberculose exige, não só investimentos em programas, diagnósticos e tratamento, mas também ações sobre os determinantes sociais, opinião corroborada por Selgelid, ${ }^{15}$ que afirma que fatores sociais desempenham papel central na epidemiologia da tuberculose, alertando sobre os cuidados em ater-se exclusivamente a intervenções farmacológicas.

Rajeswari et al. ${ }^{30}$ defendem programas de controle que consideram compreender as percepções dos pacientes, estigmas relacionados e má qualidade de vida emocional, bem como o apoio social, aconselhamento e educação em saúde, pois o aumento do conhecimento sobre a doença pode contribuir para uma visão mais positiva, tanto do paciente quanto das pessoas ao redor. ${ }^{25}$

Dhuria et al. ${ }^{31}$ fazem críticas aos programas de controle que levam em consideração a negatividade do escarro e ganho de peso como indicadores prognósticos, mas não consideram qualquer outra dimensão da saúde. Nesse sentido, ações que visem à melhoria dos fatores envolvidos, abrangendo aspectos sociais, psicológicos e físicos, bem como os determinantes da tuberculose e tratamento, são apontadas como forma de eliminar a tuberculose dentre as doenças de interesse público.

\section{Considerações finaIS}

A tuberculose é uma doença que merece atenção social e dos poderes públicos por apresentar características peculiares e de difícil controle, como a facilidade de transmissão e o tratamento prolongado que envolve uma dinâmica complexa entre a farmacoterapia e a adesão do paciente ao tratamento.

Outra característica problemática a respeito desta doença é o caráter estigmatizante que promove conflitos de relacionamento e mudanças na autoestima e que pode gerar isolamento social, transtornos no cotidiano e no âmbito profissional dos doentes.

Pelo fato de a tuberculose ser uma doença comum entre populações pobres, residentes em locais aglomerados, que apresentam condições precárias de vida e disseminada pelo mundo todo, a OMS, juntamente com outras instituições importantes, como o Ministério da Saúde, tem desenvolvido ações que visam ao controle da doença por meio de metas e objetivos, oferecendo suporte para diagnóstico e tratamento da infecção e, na atenção básica de saúde, apresentando estratégias para diminuir os casos de morte por tuberculose, principalmente em portadores do vírus HIV. Por isso, o envolvimento dos profissionais de saúde é essencial, tanto no sentido de oferecer apoio durante o tratamento quanto no que se refere ao esclarecimento de informações.

Portanto, apesar de a tuberculose ser uma doença antiga, ela ainda gera dispêndios econômicos e transtornos para a humanidade por apresentar mecanismos que conferem sua permanência entre as doenças de interesse mundial. Por isso, para alcançar as metas e objetivos para o controle da tuberculose, é necessário optar por um modelo de saúde humanizado, que articule a participação social e o envolvimento intrínseco dos profissionais de saúde e poderes públicos mundiais. 


\section{REFERÊNCIAS BIBLIOGRÁFICAS}

1. Franco C, Zanetta DMT. Tuberculose em profissionais de saúde: medidas institucionais de prevenção e controle. Arquivos de Ciências da Saúde, 2005, out.-dez; 11(4):244-52 [citado 15 ago. 2013]. Disponível em: http://www. cienciasdasaude.famerp.br/racs_ol/Vol-114/10\%20-\%20id\%2044.pdf.

2. Brasil. Ministério da Saúde. Secretaria de Vigilância em Saúde. Boletim epidemiológico: especial tuberculose, 2012; 43 [citado 2 ago. 2013]. Disponível em: http://portal.saude.gov. br/portal/arquivos/pdf/bolepi_v43_especial_tb_ correto.pdf.

3. Neville BW, Damm DD, Allen CM, Bouquot JE. Infecções bacterianas. 2a ed. Rio de Janeiro: Guanabara Koogan, 2004. Patologia oral e maxilofacial 166-68.

4. Campos HS. Etiopatogenia da tuberculose e formas clínicas. Pulmão, 2006; 15(1):2935 [citado 2 ago. 2013]. Disponível em: http://www.saudedireta.com.br/docsupload/ 13404591932.pdf.

5. Souza MVN, Vasconcelos TRA. Drugs against tuberculosis: past, present and future. Quim Nova, 2005; 28(4):678-683 [citado 20 ago. 2013]. Disponível em: http://www.scielo.br/pdf/ qn/v28n4/25117.pdf.

6. Rosemberg J. Tuberculose: aspectos históricos, realidades, seu romantismo e transculturação. Rev Saúde Pública, 1999, dez, 7(2) [citado 2 jun. 2013]. Disponível em: http://scielo.iec. pa.gov.br/scielo.php?script=sci_arttext\&pid=S0 $103460 \times 1999000200002 \&$ lng=pt.

7. Pinheiro P. Tuberculose: sintomas e tratamento. MD Saúde, 2012; [citado 2 ago. 2013]. Disponível em: http://www.mdsaude. com/2009/04/sintomas -de-tuberculose.html.

8. Sheikh S, Pallagatti S, Gupta D, Mittal A. Tuberculous osteomyelitis of mandibular condyle: a diagnostic dilemma. Dentomaxillo Fac Radiol, 2012, Feb.; 41(2):169-7. [citado 17 mar. 2014]. Disponível em: http:// www.birpublications.org/doi/full/10.1259/ $\mathrm{dmfr} / 56238546$.

9. Wang WC, Chen JY, Chen YK, Lin LM. Tuberculosis of the head and neck: a review of 20 cases. Oral Surg Oral Pathol Oral Radiol
Endod, 2009, Mar.; 107(3):381-6 [citado 17 mar. 2014]. Disponível em: http://www.ncbi.nlm. nih.gov/pubmed/19157915.

10. Organização Mundial da Saúde. Relatório Global da Tuberculose. 17a ed., 2012. [citado 2 ago. 2013]. Disponível em: http://translate. google.com.br/translate?hl=ptBR\&sl=en\& $\mathrm{u}=\mathrm{http}: / / \mathrm{www}$.OMS.int/topics/tuberculosis/ en/\&prev=/search\%3Fq\%3Dtuberculose\%2Bor ganiza $\% 25 \mathrm{C} 3 \% 25 \mathrm{~A} 7 \% 25 \mathrm{C} 3 \% 25 \mathrm{~A} 30 \% 2 \mathrm{Bmun}$ dial\%2Bsa\%25C3\%25BAde\%26hl\%3Dp-BR\% 26tbo\%3Dd\%26biw\%3D1280\%26bih\%3D636 \&sa=X\&ei=KHEBUYr6DYHs8gTEkoDoAQ\&ve $\mathrm{d}=0 \mathrm{CDwQ7gEwAQ}$.

11. Sá LD, Souza KMJ, Nunes MG, Palha PF, Nogueira JA, Villa TCS. Tuberculosis treatment in family health units: stories of abandonment. Texto Contexto Enferm, 2007, out-dez; 16(4):712-8 [citado 2 jul. 2013]. Disponível em:http://www.scielo.br/pdf/tce/v16n4/ a16v16n4.pdf.

12. Campos, HS. Mycobacteruim tuberculosis resistente: de onde vem a resistência? Bol Pneumol Sanitária, 1999; 7:51-7 [citado 2 jul. 2013]. Disponível em: http://scielo.iec.pa.gov. $\mathrm{br} / \mathrm{pdf} / \mathrm{bps} / \mathrm{v} 7 \mathrm{n} 1 / \mathrm{v} 7 \mathrm{n} 1 \mathrm{a} 06 . \mathrm{pdf}$.

13. Ruffino-Neto A. Tuberculosis: the neglected calamity. Rev Soc Bras Med Trop, 2002, jan.fev.; 35(1):51-8 [citado 1 jun. 2013]. Disponível em: http://www.scielo.br/pdf/rsbmt/v35n1/7636. pdf.

14. Rasanathan K, Kurup AS, Jaramillo E, Lonnroth $K$. The social determinants of health: key to global tuberculosis control. Int $\mathrm{J}$ Tuberc Lung Dis, 2011; 15(6):S30- S36.

15. Selgelid MJ. Ethics, tuberculosis and globalization. Public health ethics, 2008; 1(1):10-20.

16. Oliveira HB, Marín-léon L, Cardoso JC. Perfil de mortalidade de pacientes com tuberculose relacionada à comorbidade tuberculose - Aids. Rev Saúde Pública, 2004; 38(4):503-10 [citado 15 ago. 2013]. Disponível em: http://www. scielosp.org/pdf/rsp/v38n4/21078.pdf.

17. Murray PR, Rosenthal KS, Pfaller MA. Microbiologia médica. 5a ed. Rio de Janeiro: Elsevier, 2006. Capítulo 29, Mycobacterium; p. 289-302. 
18. Levinson W. Microbiologia médica e imunologia. 10a ed. Porto Alegre: Artmed, 2010. Capítulo 21, Micobactérias; p. 167-75.

19. Kumar V, Abbas AK, Fausto N. Adaptação, dano e morte celular. In: Robbins \& Cotran Patologia: bases patológicas das doenças. $7 a$ ed. Rio de Janeiro: Elsevier, 2005: p. 3-48.

20. Figueroa MCEG-S. Respuesta inmune a la infección por Mycobacterium tuberculosis: una revisión de la literatura. Rev Inst Nal Enf Resp Mex, 2001, abr.-jun.;14(2):114-28.

21. Dalcomo MP, Andrade MKN, Picon PD. Multiresistant tuberculosis in Brazil: history and control. Rev. Saúde Pública; 2007, set.; (41) suppl. 1 [citado10 ago. 2013]. Disponível em: http://www.scielo.br/pdf/rsp/v41s1/en_6570.pdf.

22. Gandhi NR, Moll A, Sturm AW, Pawinski R, Govender T, Lalloo U, et al. Extensively drugresistant tuberculosis as a cause of death in patients co-infected with tuberculosis and HIV in a rural area of south Africa. The Lancet; 2006, Oct., 4-10; 368(9547):1575-80.

23. Chakraborty MS, Chakreborty A. Tuberculosis and HIV illness. J Indian Med Assoc, 2000, Mar.; 98(3): 103-9.

24. Clementino FS, Martiniano MS, Clementino MJSM, Sousa JC, Marcolino EC, Miranda FAN. Tuberculose: desvendando conflitos pessoais e sociais. Rev Enferm Uerj, 2011, out.-dez; 19(4):638-43 [citado 15 ago. 2013]. Disponível em: http://www.facenf.uerj.br/ v19n4/v19n4a23.pdf.

25. Sengupta S, Pungrassami $P$, Balthip $Q$, Strauss $\mathrm{R}$, Kasetjaroen $\mathrm{Y}$, Chongsuvivatwong $\mathrm{V}$, et al. Social impact of tuberculosis in Southern Thailand: views from patients, care providers and the community. Int $\mathrm{J}$ Tuberc Lung Dis, 2006; 10(9):1008-12.

26. Gomes ALC, Sá LD. The concepts of bonding and the relation with tuberculosis control. Rev
Esc Enferm USP, 2009; 43(2):365-72 [citado 02 ago. 2013]. Disponível em: http://www.scielo.br/ pdf/reeusp/v43n2/en_a16v43n2.pdf.

27. Ruffino-neto, A. Tuberculosis in Brazil: general information and news perspectives. Inf Epidemiol SUS, 2001, set.; 10(3):129-38 [citado 10 mar.2013]. Disponível em: http://scielo.iec. pa.gov.br/pdf/iesus/v10n3/v10n3a04.pdf.

28. Hargreaves JR, Boccia D, Evans CA, Adato $M$, Petticrew M, Porter JDH. The social determinants of tuberculosis: from evidence to action. Am J Public Health, 2011, Apr.; 101(4): 654-62.

29. Nogueira JA, Sá LD, França UM, Almeida SA, Lima DS, Figueiredo TMRM, et al. The information system and tuberculosis control on prioritary cities of Paraiba - Brazil. Rev Esc Enferm USP, 2009; 43(1):125-31 [citado 10 mar. 2013]. Disponível em: http://www.scielo. $\mathrm{br} / \mathrm{pdf} /$ reeusp/v43n1/en_16.pdf.

30. Rajeswari R, Muniyandi M, Balasubramanian R. Perceptions of tuberculosis patients about their physical, mental and social well-being: a field report from South India. Soc Sci Med, 2005, Apr.;60(8):1845-53.

31. Dhuria M, Sharma N, Ingle GK. Impact of tuberculosis on the quality of life. Indian $\mathrm{J}$ Community Med, 2008, Jan.; 33(1):58-9.

Érika Felix da Silva Santos.

Aluna Graduanda do curso de Odontologia da Universidade Estadual da Paraíba. Campina Grande/PB, Brasil.: erika_felix2012@hotmail.com. Endereço para correspondência: Érika Felix da Silva Santos. Rua Pedro Leão, 190, Catolé. CEP: 58410-133.

Submetido em: 15-10-2013

Aceito em: 13-5-2014 